\title{
Image Information Mining Utilizing Hierarchical Segmentation
}

\author{
James C. Tilton \\ NASA's Goddard Space \\ Flight Center \\ Mail Code 935 \\ Greenbelt, MD 20771 USA \\ James.C.Tilton.1@gsfc.nasa.gov
}

\author{
Giovanni Marchisio and \\ Krzysztof Koperski \\ Insightful Corporation \\ 1700 Westlake Ave. N, Ste. 500 \\ Seattle, WA 98109 USA \\ giovanni@insightful.com \\ krisk@insightful.com
}

\author{
Mihai Datcu \\ German Aerospace Center \\ - DLR \\ Oberpfafenhofen \\ WeBling, GERMANY \\ mihai.datcu@dlr.de
}

\begin{abstract}
The Hierarchical Segmentation (HSEG) algorithm is an approach for producing high quality, hierarchically related image segmentations. The VisiMine image information mining system utilizes clustering and segmentation algorithms for reducing visual information in multispectral images to a manageable size. The project discussed herein seeks to enhance the VisiMine system through incorporating hierarchical segmentations from HSEG into the VisiMine system.
\end{abstract}

\section{INTRODUCTION}

The Hierarchical Segmentation (HSEG) algorithm is an approach for producing high quality, hierarchically related image segmentations. The HSEG algorithm is a hybrid of region growing and spectral clustering that produces a hierarchical set of image segmentations.

Different image analysis applications often require different levels of image segmentation detail. Further, a particular application may require different levels of image segmentation detail depending on the image objects being segmented. Thus, a choice of several levels of image segmentation detail is often very useful. The HSEG algorithm automatically produces a hierarchical set of segmentations, based on detected convergence points. An analyst can select between segmentations at different levels in the segmentation hierarchy to produce the most appropriate segmentation for a particular application.

The VisiMine image information mining system utilizes clustering and segmentation algorithms for reducing visual information in multispectral images to a manageable size. The indexing strategy extracts three levels of features: 1) pixel level, 2) polygon level, and 3) tile level features. The pixel level features are used for the extraction of higher-level features. Fast segmentation algorithms based on PDE methods are used to decompose image tiles into polygons.

In the paper we compare the results of similarity searches utilizing features extracted using an enhanced version of CLARA algorithm (Clustering LARge Applications) in combination with statistical sampling with the results that use hierarchical image segmentation (HSEG) to derive class

This project is supported by NASA's Intelligent Systems Program, NASA Headquarters Code $R$ boundaries for land cover. We present the results of polygon and tile similarity searches based on spectral and textural properties. The use of HSEG features improves the precision and recall of similarity searches. The interactive label training and retrieval based on Bayesian models also benefits from hierarchical image segmentation approach.

\section{HIERARCHICAL SEgmeNTATION}

We provide here a high level description of the HSEG algorithm. This description is a refinement of the description given earlier in [1] and follows that given in [2]:

\section{HSEG Basic Algorithm Description:}

1. Give each image pixel a region label and set the global criterion value, critval, equal to zero. If a presegmentation is provided, label each image pixel according to the pre-segmentation. Otherwise, label each image pixel as a separate region.

2. Calculate the dissimilarity criterion value between each spatially adjacent region.

3. Find the smallest dissimilarity criterion value and merge all pairs of spatially adjacent regions with this criterion value.

4. Calculate the dissimilarity criterion value between all pairs of non-spatially adjacent regions (optional). Multiply these dissimilarity criterion values by spclust_wght (a preset weighting factor with default value 1.0).

5. Merge all pairs of non-spatially adjacent regions with dissimilarity criterion value less than or equal to the criterion value found in step 3 (optional).

6. If the number of regions remaining is less than the preset value chkregions, go to step 7 . Otherwise, go to step 2 .

7. Let prevcritval $=$ critval. Calculate the current global criterion value and set critual equal to this value. If prevcritval $=$ zero, go to step 2 . Otherwise calculate cvratio = critual/prevcritual. If cvratio is greater than the preset threshold convfact, save the region label map from the previous iteration as a "raw" segmentation result. Also, store the region number of pixels list, region mean vector list and region criterion value list for this previous iteration. (Note: The region criterion value is the portion of the global criterion value contributed by 
the image pixels covered by the region.) If the number of regions remaining is two or less, save the region label map from the current iteration as the coarsest instance of the final hierarchical segmentation result and stop. Otherwise, go to step 2.

The results stored in step 7 can be used in post-processing analysis to select a single segmentation from the segmentation hierarchy using an analysis tool such as the "Region Labeling Tool" described elsewhere [3]. In this paper we explore incorporating the hierarchical segmentations into the VisiMine image information mining system.

A practical implementation of the HSEG algorithm requires either recursion or a coarse-to-fine spatial resolution processing scheme. Large-scale parallel processing is required to obtain reasonable processing times for either implementation scheme. Further, a "pixel switching" scheme must be employed in the recursive implementation in order avoid processing window artifacts, and a "pixel splitting" scheme must be employed in the coarse-to-fine approach when moving to finer spatial resolutions. A future paper will address these implementation issues.

\section{VISIMINE IMAGE INFORMATION MINING}

VisiMine project aims to provide the infrastructure and methodology required for the analysis of satellite images. In order to facilitate the analysis of large amounts of image data, we extract features of the images. Large images are partitioned into a number of smaller, more manageable image tiles. In addition to providing faster extraction of segments, partitioning allows fetching of just the relevant tiles when retrieval of only part of the image is requested. Then those individual image tiles are processed to extract the feature vectors. The VisiMine architecture supports three levels of features:

1. pixel level features,

2. region level features, and

3. tile level features.

The feature extraction process starts with the analysis of spectral and textural properties at the pixel level. The numerical pixel data can be clustered in order to find a small number of classes. At the same time, the tile level features may be extracted, thereby creating histograms of the pixel classes for each tile.

The extraction of region level features starts with a segmentation based on the algorithm presented in [4]. This function segments an input image into non-overlapping regions by minimizing an energy functional that trades off the similarity of regions against the length of their shared boundary. The geometrical properties of regions, such as image moments, are extracted. Based of the pixel features, the system creates histograms, mean. and standard deviation features for each region.
Additional features are extracted using raster information, such as digital elevation maps. These features can be created at all three levels.

\section{A. Feature clustering using CLARA}

In order to build features that are used in the information retrieval and data mining functions, we perform unsupervised clustering using the CLARA algorithm [5]. The user chooses the number of classes, and each pixel is assigned to a single class. Textural information may be processed in the same way to extract textural classes. For tile and region level features, histograms of pixel distributions between classes are created.

\section{B. Texture Feature Extraction}

We extract pixel level texture features by using Gabor wavelets. In our comparison study of texture based classification, the Gabor features were judged to provide superior performance when compared to other texture analysis methods, such as edge attribute processing methods, circular simultaneous autoregressive model methods, or hidden Markov model methods [6]. In VisiMine, for each pixel we extract eight texture features $\left.\alpha_{i}\right|_{i=0,7}$ by using Gabor Filters with kernels rotated by $i \pi / 8$. To obtain rotation invariant features, we find the values of the autocorrelation function [6]:

$$
t_{n}=\sum_{i=0}^{7}\left|a_{i}\right|\left|a_{(i+\pi) \bmod 8}\right|
$$

To minimize the range of the pixel index, we have chosen to compute values of the autocorrelation for $n=0,4$. These values correspond to the $0^{\circ}$ and $90^{\circ}$ differences in the orientation of the Gabor kernels. This shift should allow detection of urban road networks, which usually are correlated within a $90^{\circ}$ rotation of the wavelet kernels. The texture features are extracted using two different scales. Rotation invariance can be observed, and urban regions are judged to be similar, regardless of the orientation of street networks. For example, the suburban area of New Westminster, in the Greater Vancouver area, is judged to be similar to East Vancouver, despite the fact that the principal directions of the street networks differ by about $30^{\circ}$ for these two regions. The extraction of other microfeatures, such as frequency and orientation, also is possible [7], and we plan to perform more experiments with these features in the future.

In addition to the extraction of Gabor texture features, the system provides for extraction of Haralick's coocurrence features and Laws texture features. But, in our information retrieval experiments, we found the Gabor textures to be superior in terms of recall and precision levels. 


\section{Similarity Search}

VisiMine uses an SQL-like query language that enables specification of the data mining task, the features that are to be used in the mining process, and any additional constraints. The system is capable of performing similarity searches based on any combination of features. A user can look for the most similar image tiles, or for the most similar regions based on a pattern tile or a pattern region. VisiMine allows arbitrary weighting of the features. The values of the features can be adjusted to have the range $[0,1]$, they can be multiplied by a specific value, or they can remain the same.

\section{RESULTS}

In our experimentation with region based searches we looked only for regions having areas larger than 3000 pixels. The feature values were scaled to the range $[0,1]$. For CLARA algorithm we used 25 clusters, which warranties good results for this algorithm. The number of clusters for the RHSEG algorithm varied based on the level. For the experiments we used only histograms based on clusters that contained more than 1000 pixels from $4096 \times 4096$ Landsat TM image from over the Washington, DC/Baltimore, MD area. We compared the results of similarity searches based on CLARA features with searches based on HSEG features, or on a combination of the these features with Gabor texture features.

In the oral presentation we will present detailed results using tables and graphs. Unfortunately, space requirements do not allow inclusion of a complete set of detailed results in the proceedings paper. An example of such results is provided here in Table 1.

The best results are obtained using level 1 and 2 of HSEG and adding texture features improves the results of the majority of the queries. The best results depend on the type of the query. We believe that using the relevance feedback for the automated selection of the subsets of the features should improve the accuracy of search. In the future we are planning to use the relevance feedback for automated selection of the subsets of the features should improve the accuracy of search.

\section{FUTURE WORK}

In the future, we are planning to use the relevance feedback for automated selection of the subsets of the features should improve the accuracy of search. We will also investigate methods for automatically and adaptively selecting the hierarchical segmentation levels utilized.

The results of information retrieval can be improved using the Synthetic Aperture Radar (SAR) data. We are planning to perform feature extraction and classification/mining examples based on SRTM data for the Baltimore area. We also want to compare the topology of the HSEG intermediate outputs for scenes at two different times for the same area. The preliminary analysis shows the patterns that reinforce the classification process and help identify outliers in the data.

\section{REFERENCES}

[1] J. C. Tilton, "A recursive PVM implementation of an image segmentation algorithm with performance comparisons between the HIVE and the Cray T3E." Proceedings of the Seventh Symposium on the Frontiers of Massively Parallel Computation (Frontiers '99), Annapolis, MD pp. 146-153, Feb. 21-25, 1999.

[2] J. C. Tilton, "Method for Recursive Hierarchical Segmentation combining Greedy and Hierarchical Stepwise Optimal Approaches and Region Splitting," Disclosure of Invention and New Technology (Including Software): NASA Case No. GSC 14,474-1, NASA's Goddard Space Flight Center, April 19, 2001.

[3] J. C. Tilton, "A Region Labeling Tool for use with Hierarchical Segmentation," Disclosure of Invention and New Technology (Including Software): NASA Case No. GSC 14,331-1, NASA's Goddard Space Flight Center, February 29, 2000.

[4] Koepfler, G., C. Lopez and J. M. Morel, A Multiscale Algorithm for Image Segmentation by Variational Method, SIAM Journal of Numerical Analysis, vol. 31, pp. 282 - 299, 1994.

[5] Kaufman, L. and P. J. Rousseeuw. Finding Groups in Data: An Introduction to Cluster Analysis. Wiley, New York, 1990.

[6] Hayley, G. M., and B. M. Manjunath, Rotation Invariant Texture Classification using Modified Gabor Filters, In Proc. of IEEE ICIP95, pp. 262 - 265, 1994.

[7] Fountain, S. R., T. N. Tan, K. D. Baker. A Comparative Study of Rotation Invariant Classification and Retrieval of Texture Images. In On-Line Proceedings of the Ninth British Machine Vision Conference 1998. http://www.bmva.ac.uk/bmvc/1998/index.htm.

TABLE I

SIMILARITY SEARCH RESULTS USING VARIOUS FEATURES

\begin{tabular}{|c|c|}
\hline Feature & Average Precision \\
\hline 25 spectral CLARA clusters & 0.7522999 \\
\hline $\begin{array}{c}\text { 25 spectral CLARA clusters } \\
\text { with 10 Gabor texture clusters }\end{array}$ & 0.7304257 \\
\hline HSEG level 1 region histograms & 0.7746878 \\
\hline HSEG level 2 region histograms & 0.7728281 \\
\hline $\begin{array}{c}\text { HSEG level 2 clusters } \\
\text { with 10 Gabor texture clusters }\end{array}$ & 0.7649297 \\
\hline HSEG level 3 region histograms & 0.7207362 \\
\hline $\begin{array}{c}\text { HSEG level 3 clusters } \\
\text { with 10 Gabor texture clusters }\end{array}$ & 0.7528406 \\
\hline HSEG level 4 region histograms & 0.7061142 \\
\hline HSEG level 6 region histograms & 0.6435331 \\
\hline HSEG level 7 region histograms & 0.4792615 \\
\hline
\end{tabular}

